\title{
PULMONARY MANIFESTATIONS OF RHEUMATOID ARTHRITIS IN ALGERIAN POPULTION
}

\author{
S. Lefkir - Tafiani , A. Nebab , B. Bengana, N. Brahimi , A. Ladjouze \\ University hospital of Benimessous, Rheumatology department, Algiers, Algeria. \\ University hospital of Bab Eloued, Epidemiology departement, Algiers, Algeria \\ University hospital of Benaknoune, Algiers, Algeria
}

Introduction: Rheumatoid arthritis (RA) is the most common chronic inflammatory disease. Considered as a connective tissue disease, it can be complicated by extra-articular and systemic manifestations, especially pulmonary involvement characterized by its frequency and severity.

Usually it occurs several pulmonary injuries with the presence of pulmonary nodules, which causes $10 \%$ to $20 \%$ of mortality in these cases. The advent of high resolution computed tomography (CT) has led to the detection of subclinical and infraradiographic lesions.
Our study aims are :

To estimate the prevalence of pulmonary involvement in Algerian population with RA

To describe the clinical-biological and radiological characteristics of different pulmonary injuries .

To identify the link with a high level of anti-citrullinated peptide antibodies (ACPA) .

Methods and patients: It's a multicenter and descriptive cross-sectional study.

We have collected a demographic and clinical radiological data for patients over 16 years meeting the criteria of ACR / EULAR (2010) for RA with verified pulmonary damages between January 2013 and June 2016

We have excluded all patients with particularly opportunistic infections and lung cancers.

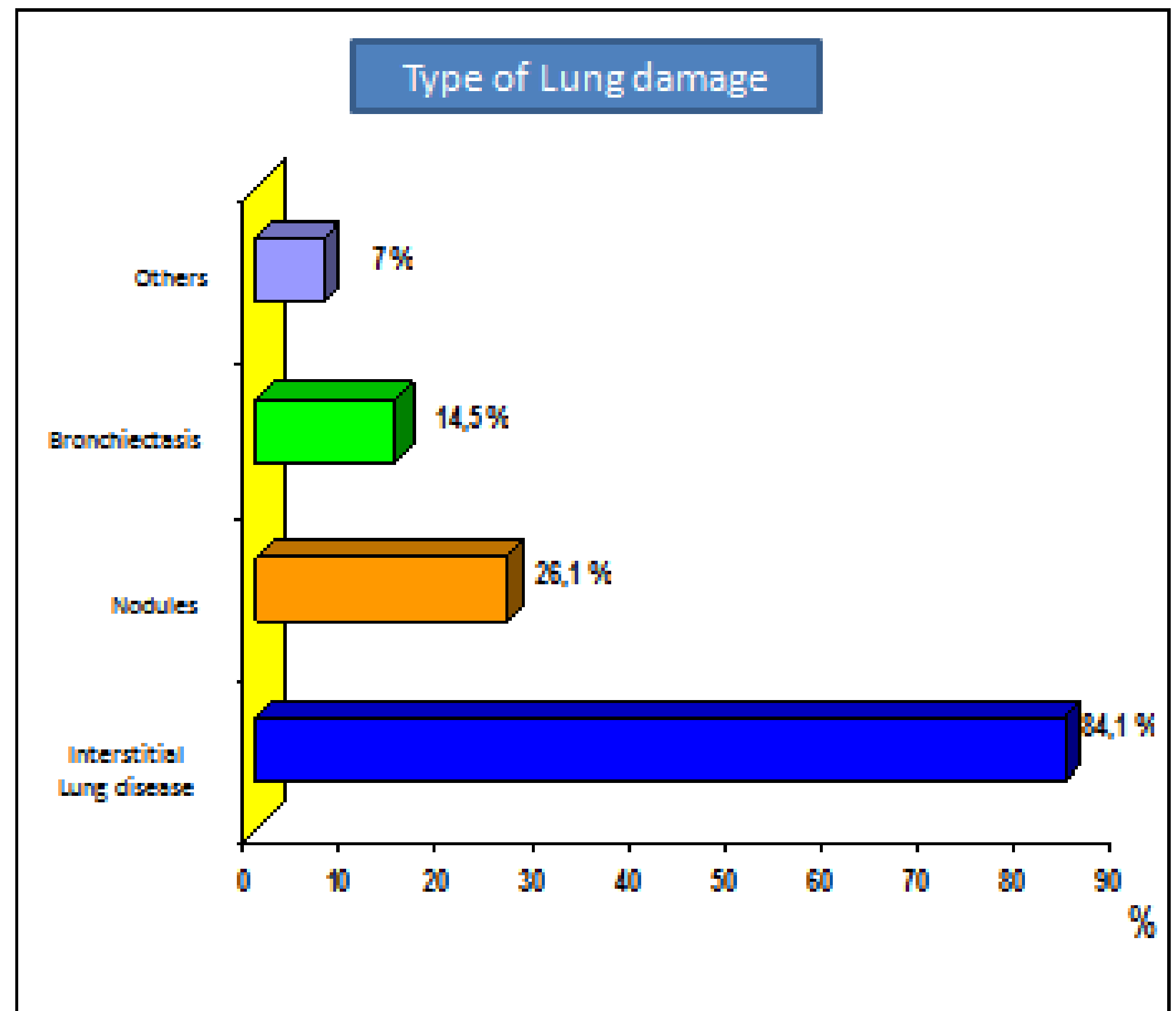

Results: We have included 69 patients, 20 were former cigarette smokers. Majority of subjects were women. Different pulmonary phenotypes were identified: Various types of ILD (84\%), pulmonary nodules (26\%), small airways disease (10\%): as bronchiolitis and bronchiectasis. We noted $63 \%$ of manifestations with high-titer of anti-CCP but there was no difference in both groups: with or without antiCCP $(p=0.35)$. Multivariable analysis revealed a positive relationship between age of disease (odds ratio $[\mathrm{OR}], 2.33$; 95\% confidence interval $[\mathrm{Cl}])$, and smoking in males $(\mathrm{OR}, 2.72-4.70,95 \% \mathrm{Cl}$ ) with severity of lung diseases in RA

\begin{tabular}{|l|c|c|}
\hline Personnal history & $\mathbf{N}$ & $\mathbf{\%}$ \\
\hline Actual smoking & 20 & $29 \%$ \\
\hline Passive smoking & 6 & $8.7 \%$ \\
\hline Exposure to silica & 1 & $1.4 \%$ \\
\hline Others exposures & 2 & $2.9 \%$ \\
\hline
\end{tabular}

\section{Distribution of patients by sex}

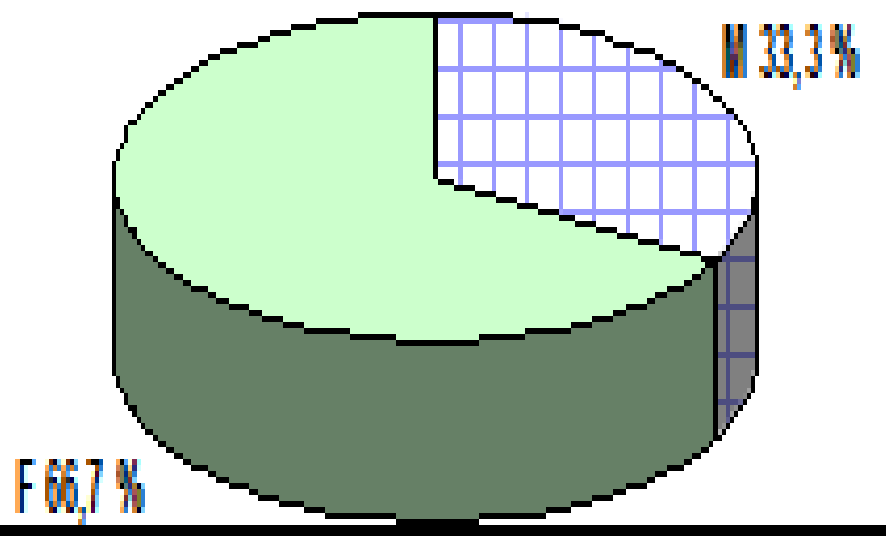

Interstitial lung disease is the most common type of disease in the RA population.

More than half of RA were erosive and more than $2 / 3$ were with ACPA.

The average age of patients is 57.26 years, the standard deviation is 12 years with an IC $=$ [54.38 years -60.15 years].

Conclusion: The presence of a high level of anti-CCP in RA does not seem to be more related to the rheumatoid lung, however the risk seems significant if the RA is old and if chronic smoking is associated with it.

Prospective studies on larger samples are needed to assess the link The sex ratio is $46 / 23=2$ (two women for a man) between anti CCP and lung damage. 